\title{
A General-Purpose Mobile Framework for Situated Learning Services on PDA
}

\author{
Seong Baeg Kim, Kyoung Mi Yang, and Cheol Min Kim \\ Department of Computer Education, Cheju National University, \\ 66 Jejudaehakno, Jeju-si, Jeju-do, 690-756, Korea \\ \{sbkim, kmyang, cmkim\} @cheju.ac.kr
}

\begin{abstract}
Recently, as ubiquitous technology has been developed rapidly, a specific application service considering users' situations has been becoming the focus of attention. As a field of a typical situation-based application, we focus on situated learning using a mobile device. A u-learning system can provide learners with individualized learning at any time in any place. In this paper, we propose a general-purpose mobile framework suitable for situated learning on PDA with RFID mobile system. Specially, we examine three aspects in making the framework. First, we investigate how learners obtain and access customized learning contents suitable for them. Second, we construct a mobile middleware for RFID on PDA. Finally, we devise the application-specific system for situated learning.
\end{abstract}

Keywords: RFID, Situated Learning, Mobile middleware, Framework.

\section{Introduction}

In recent years, the development of information technology has encouraged the spread of various mobile devices. Thus, the attention to a ubiquitous system has been increasing. In general, a u-learning using a mobile device is more suitable for customized learning. Furthermore a u-learning can be used to bring situated learning for each learner. It has been recognized that web-based education systems enable learners to have self-directed learning. However, they have still limits on making possible customized learning reflecting differences such as degrees of knowledge, learning capability and learning goals. For this reason, the systems have activities made based just on inexact learning preferences of the learner and without doubt aren't supporting effectively the learner for active and individualized learning.

There has been research regarding customized learning for each learner $[1][2][4][5]$. However, there has been little research on personalized learning considering situation. Also, the previous research on middleware system has focused on constructing a middleware system on a desktop or server [3]. However, it is also essential to construct a mobile middleware platform for developing the mobile applications. The platform would be small volume, which includes minimal APIs required for developing an application. From the viewpoint of situated learning theory [7], the learning effect of using traditional learning methods is not good, because it are mostly failed for learners to apply the knowledge in an actual situation that is 
obtained from learning without consideration of an actual situation. To solve it, the situated learning theory has been studied [7].

\section{Profiling Scheme}

The profile data collected from a mobile device are sent using an encoding scheme the form of the variable HTTP_USER_AGENT in order to provide customized learning based on situation. Fields composed of the encoded values are divided into 4 main parts:PDA Profile, Learner Information, GPS Information and RFID Information. They are split by a semicolon.

We encoded the values of the device manufacturer field with 15 mobile device makers we have studied so far. When known the manufacturer and model of a PDA, such hardware specifications of the PDA as CPU, RAM, and ROM etc. can be extracted from a server. This information would be used in providing the customized learning at right time in right place on the PDA that each learner has. Learner information is composed of learner's age, learning time, learning subject, learning type, and learning motive.

In case of GPS information, it enables the system to find the exact location and the moving direction of the learner at a certain time. Also, in case of RFID information, it enables the system to identify an exact situation given. We encoded the RFID information based on EPC (Electronic Product Code) that is currently used in popular. The standard EPC consists of header, EPC manager, object class, and serial number [6].

\section{Mobile RFID Middleware}

In addition to our profiling system, there is a mobile RFID middleware developed in order to support situated learning based on RFID. The structure of the mobile RFID

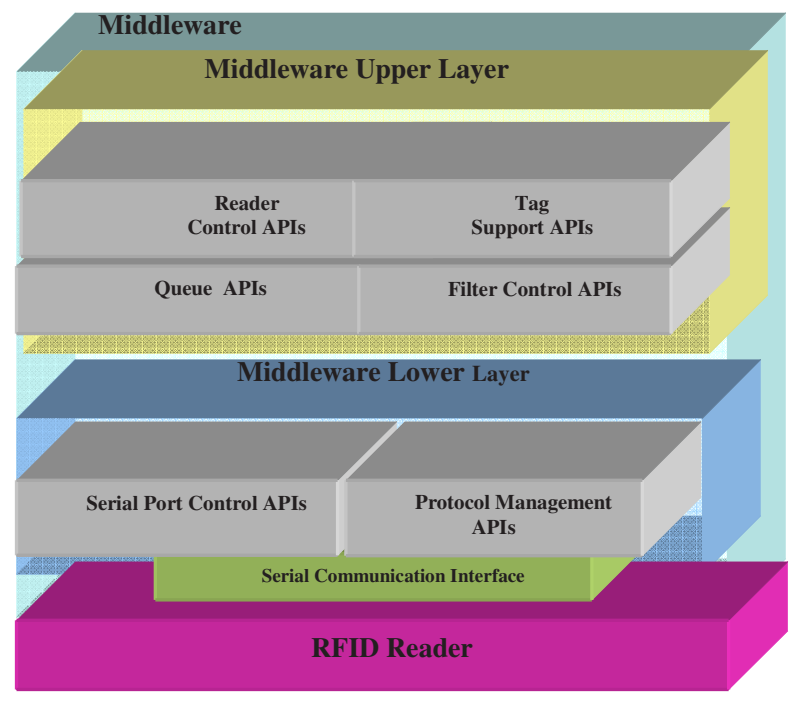

Fig. 1. The Structure of the Mobile RFID Middleware 
middleware is shown in Figure 1. The middleware consists of two layers: upper layer, lower layer. The upper layer provides APIs for supporting applications. The lower layer has functions for various RFID readers. The APIs of the lower layer consists of the serial port control APIs and the protocol management APIs.

\section{Situated Learning}

The situated learning theory based on constructivism explains that learning can be effective when knowledge is provided in the meaningful context. The overall architecture for situated learning on PDA is shown in Figure 2. The application
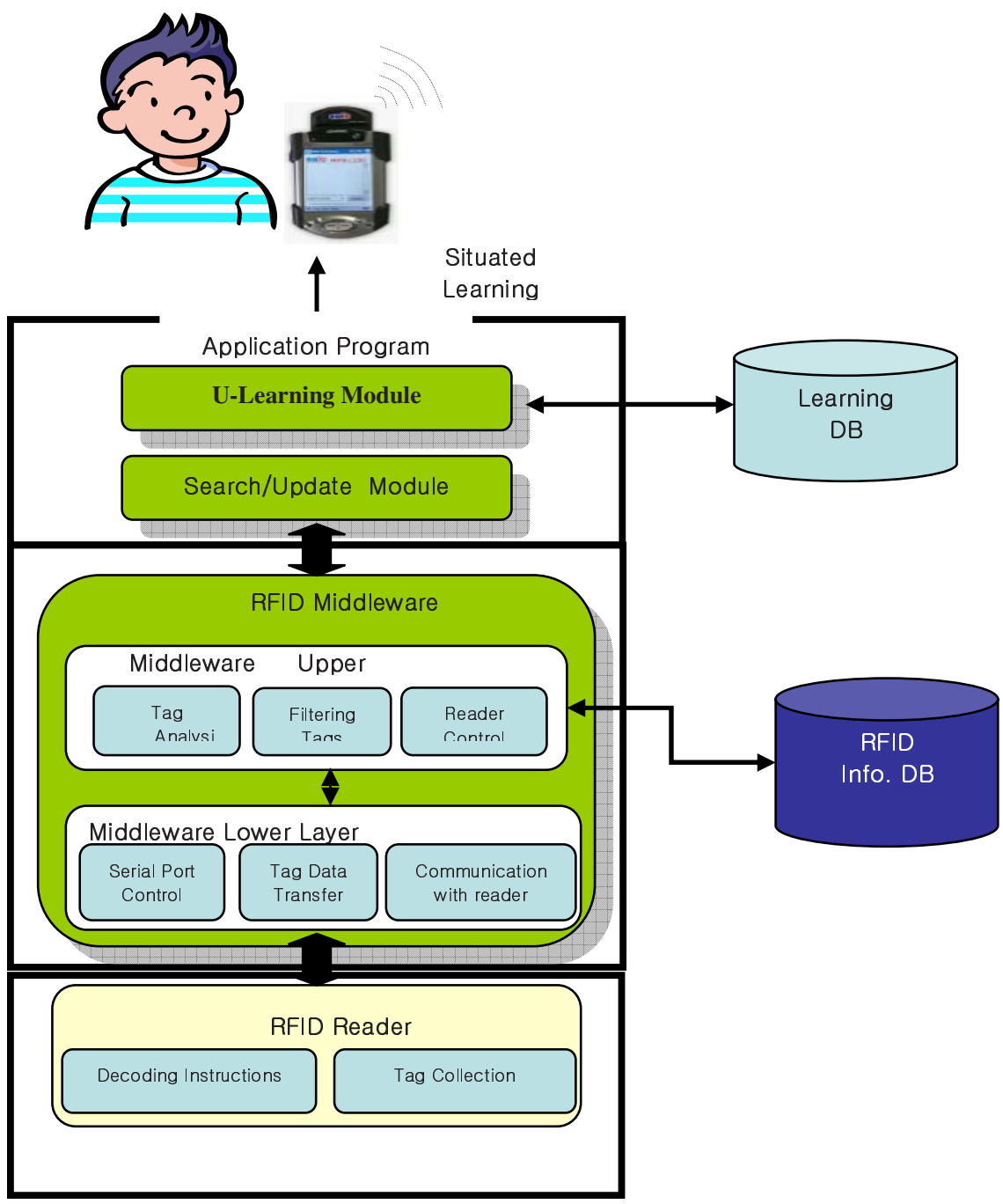

Fig. 2. The Overall Structure for Situated Learning 
program consists of U-learning module and Search/Update module. U-learning module is implemented using U-learning APIs. Search/Update module provides the function to search and update databases that serve RFID and learning information.

\section{Conclusion}

We suggested a general-purpose mobile framework for enabling situated learning services under ubiquitous environment. In the framework, we described how to encode learner's profiling information considering the characteristics of a mobile device and each learner, which is required for providing personalized learning at the right time in the right place. That is to come up with a way of providing a best-suited learning environment for each learner who uses a mobile device. Also, we proposed the mobile RFID middleware, which is largely composed of two parts:upper layer and lower layer. Finally, we presented the overall structure of application for situated learning using RFID reader, which is attached to a mobile device PDA.

We're optimizing the mobile RFID middleware modules and tuning it to improve performance and reliability.

\section{References}

1. Tan-Hsu Tan, Tsung-Yu Liu: The Mobile-Based Interactive Learning Environment (MOBILE) and A Case Study for Assisting Elementary School English Learning", In Proceedings of the $4^{\text {th }}$ IEEE International Conference on Advanced Learning Technologies, (2004).

2. Phivos Mylonas, Paraskevi Tzouveli, Stefanos Kollia: Towards a Personalized e-learning scheme for Teachers, In Proceedings of the $4^{\text {th }}$ IEEE International Conference on Advanced Learning Technologies, (2004).

3. T. Jung, Y. Kim, Y. Lee: The Platform Technology of RFID Middleware, Telecommunications Review, Vol. 15, No. 2, (2005).

4. Abbattista, F., Degemmis, M., Fanizzi, N., Licchelli, O., Lops, P., Semeraro, G., and Zambetta, F.: Learning User Profiles for Content-Based Filtering in e-Commerce, In Proceedings of AI Workshop su Apprendimento Automatico: Metodi e Applicazioni. (2002).

5. Ricardo Carreira, Jaime M. Crato,Daniel Gonçalves, Joaquim A Jorge: Evaluating Adaptive User Profiles for News Classification, In proceedings of IUI'04, (2004).

6. S. Sarma, D. Brock, and D. Engels: Radio Frequency Identification and the Electronic Product Code, IEEE Micro, Vol. 21, (2001).

7. S. Kim: Good Ideas to Foment Educational Revolution, Communications of Korea Educational Technology Research Association, Vol. 38, No. 1, (1998). 